\title{
El comportamiento del sensor electromagnético y su calibración frente a la salinidad edáfica
}

\author{
D López-Bruna, J Herrero * \\ Diputación General de Aragón, Servicio de Investigación Agraria, Apartado 727, E50080 Zaragoza, España
}

(Reçu le 17 novembre 1994 ; accepté le 15 janvier 1995)

\begin{abstract}
Resumen - Las técnicas electromagnéticas (EM) permiten cartografiar la salinidad edáfica con sensor portátil. Pero las lecturas del sensor deben traducirse, por calibración, a una medida estándar como la conductividad eléctrica del extracto saturado (CEe). Algunos autores usan transformaciones no lineales para mejorar las calibraciones. En este trabajo se utilizan muestras de suelo hasta $1 \mathrm{~m}$ de profundidad tomadas en tres años consecutivos en une parcela de regadio, mostrando que los métodos lineales son suficientemente precisos para cartografiar la salinidad del suelo. Además, parecen ofrecer información sobre el perfil de salinidad, si bien este aspecto deberá estudiarse más detenidamente. Las caractérísticas técnicas del sensor EM empleado producen una relación no lineal entre la conductividad eléctrica del suelo y la lectura del sensor, que puede corregirse en base a los datos técnicos facilitados por el fabricante.
\end{abstract}

suelo / salinidad / sensor electromagnético / riego

Résumé - Le comportement du senseur électromagnétique et sa calibration vis-à-vis de la salinité du sol. Les techniques électromagnétiques (EM) permettent de cartographier la salinité des sols avec un senseur portatif. Mais les lectures du senseur doivent être traduites, par calibration, en une mesure standard comme la conductibilité électrique de l'extrait saturé (CEe). Certains auteurs utilisent des transformations non linéaires pour améliorer les calibrations. Dans le présent travail, on a utilisé des échantillons de sol jusqu'à $1 \mathrm{~m}$ de profondeur prélevés trois années successives dans une parcelle irriguée, ce qui a permis de montrer que les méthodes linéaires sont suffisamment précises pour cartographier la salinité du sol. De plus, elles semblent offrir de l'information sur le profil de salinité, encore que cet aspect doivent être encore étudié de manière plus approfondie. Les caractéristiques techniques du senseur EM utilisé produisent une relation non linéaire entre la conductivité électrique du sol et la lecture du senseur, qui peut être corrigée sur la base des données techniques fournies par le fabricant.

sol / salinité / senseur electromagnétique / irrigation

Summary - The behaviour of the electromagnetic sensor and its calibration for soil salinity. Soil salinity survey is easy with a hand-held electromagnetic sensor (EM), but the readings have to be converted into a standard measure of soil salinity, such as the electrical conductivity of the soil saturated extract (CEe). Several authors use nonlinear transformations of EM readings to avoid collinearity between regression variables or to decrease the errors of the estimates. EM data and $1-m$-depth soil samples taken on an irrigated plot in three consecutive years, showed that linear methods of calibration are sufficiently accurate for soil salinity surveys. Linear methods also seem to give information 
about the salinity profile, although this aspect should be tested further. Nonlinearities between EM readings and bulk soil electrical conductivity may appear due to the design characteristics of the EM sensor employed, but they can be corrected for using the technical characteristics provided by the manufacturer.

soil / salinity / electromagnetic sensor / irrigation

\section{INTRODUCCION}

La medida in situ de la salinidad edáfica mediante inducción electromagnética se ha hecho viable gracias al desarrollo de sensores portátiles, capaces de dar medidas en campo con gran rapidez. El uso y los principios de estos aparatos han sido descritos por McNeill 1980a, 1980b. Básicamente consisten en dos bobinas paralelas, separadas por una determinada distancia; una emite una onda electromagnética y la otra actúa como receptora. Al propagarse dicha onda en une medio conductor de la electricidad, como lo es un suelo salino y/o húmedo, la señal en la bobina receptora difiere de lo que sería en el vacío. Cuando se cumplen las condiciones de operación señaladas por el fabricante, la comparación de las señales emitida y recibida informa acerca del medio. Un sensor de salinidad edáfica ha de ser sensible preferentemente a la profundidad de la capa radicular, y a valores de CE en el intervalo de las encontradas en los suelos.

El sensor electromagnético (SEM) utilizado en este trabajo es el Geonics EM-38, diseñado para medir la conductividad eléctrica del suelo si éste es homogéneo o, por lo menos, estratificado en capas de conductividad eléctrica constante, y si dicha conductividad no excede un umbral en torno a $10 \mathrm{dS} / \mathrm{m}$ (Mc Neill, 1980a). Sin embargo, no resultaría útil restringir el uso del SEM a condiciones de homogeneidad que, además, son dificilmente constatables en el campo. En cuanto a la conductividad, autores como Mc Neill (1980a, 1980b) y Cook (1992) se refieren a la condición de 'bajo número de inducción', es decir, valores de la conductividad para los que la respuesta del sensor puede considerarse lineal. Si bien McNeill (1980a) ofrece una expresión que relaciona la conductividad eléctrica del suelo con la intensidad de los campos en las bobinas emisora y receptora del SEM, no se ha encontrado en la literatura una expresión teórica que ofrezca las lecturas del sensor como función explícita de la conductividad eléctrica del medio prospectado. Al respecto, Corwin y Rhoades (1990) presentan una tabla con valores de la conductividad de un medio homogéneo y las correspondientes lecturas del SEM (EM-38) para 15 valores en el inter- valo $0-10 \mathrm{dS} / \mathrm{m}$, pero no indican el modo de obtención de dicha tabla.

Un problema clásico de los métodos de inducción electromagnética para medir la salinidad es la conversión de las lecturas del sensor a los parámetros más comunes en edafología, como son la conductividad eléctrica (CE) de distintos extractos de suelo que, además, corresponden a intervalos de profundidad del suelo determinados. Con este propósito se han desarrollado diversos métodos de calibración que relacionan la lectura del sensor con la CE media con sonda de cuatro electrodos (Corwin y Rhoades, 1982, 1984), o con la CE del extracto de saturación (Slavich y Petterson, 1990; Lesch y Rhoades, 1992; Díaz y Herrero, 1992).

El seguimiento de la evolución de la salinidad edáfica en los regadiós del Viejo Mundo, a menudo con parcelas pequeñas y gran variedad de suelos dentro del mismo regadío, requiere poner a punto métodos simples de calibración del SEM para cada parcela. En tal sentido, este artículo no utiliza procedimientos estadísticos complejos ni grandes cantidades de muestras de suelo o de lecturas de SEM, sino que partiendo de las características físicas del SEM discute la interpretación de los datos obtenibles asumiendo las limitaciones prácticas del trabajo en campo, sobre parcelas "no experimentales", es decir cultivadas por sus propietarios en condiciones de rutina. Por otra parte, la calibración alcanzada sólo se propone como válida para la propia parcela, ello exime de un estudio de características de suelo que deberian tomarse en cuenta si se pretendiera generalizar tal calibración.

Los objetivos de este trabajo son (a) contrastar tres métodos de calibración del EM-38 con la conductividad eléctrica del extracto de saturación (CEe), y (b) explicitar la relación teórica entre la CE del suelo y las lecturas del EM-38 para facilitar las calibraciones.

\section{TEORÍA}

Los sensores electromagnéticos comercializados por Geonics Ltd responden a las características indicadas por McNeill (1980a), que pueden parti- 
cularizarse a cada modelo conociendo su frecuencia de trabajo y la distancia entre bobinas. En el caso del EM-38 ambos parámetros son fijos (13,2 KHz y $1 \mathrm{~m}$ respectivamente), dependiendo la lectura del modo en que se orienten los ejes de las bobinas emisora y receptora (paralelas entre sí) con respecto al plano del suelo. Así, según dicho autor, un horizonte de pequeño grosor $\Delta z$ que se encontrara a una profundidad $z$ en el suelo y tuviera una conductividad constante $\sigma$, contribuiría a la lectura con un valor $\Phi_{H} \sigma \Delta z$ si el sensor se dispusiera horizontalmente en la superficie, y $\Phi_{\bigvee} \sigma \Delta z$ si verticalmente, donde:

$$
\begin{aligned}
& \Phi_{H}=2-4 z\left(4 z^{2}+1\right)^{-1 / 2} \\
& \Phi_{V}=4 z\left(4 z^{2}+1\right)^{-3 / 2}
\end{aligned}
$$

de modo que si se tiene un perfil de conductividades continuo $\sigma=\sigma(\mathrm{z})$ en profundidad, la lectura del SEM en cualquiera de los dos modos es la suma ponderada de todas las capas; en el límite:

$$
E M=\int \Phi(z) \sigma(z) d z
$$

donde EM simbolizaOEMH o EMV, según se sustituya $\Phi(z)$ por [1a] o [1b]

El alcance teórico es infinito, es decir, todas las capas contribuyen con su conductividad, por lo cual a la lectura [2] siempre se aportarán cantidades no contrastadas con medidas de laboratorio sobre muestras de suelo. Sin embargo, aplicando [2] y supuesta una misma salinidad en todo el perfil, las capas que afectan en menos de un $10 \%$ a la lectura en posición vertical (EMV) se encuentran por debajo de $5 \mathrm{~m} \mathrm{y}$, análogamente, por debajo de 2,5 $\mathrm{m}$ para la lectura en posición horizontal (EMH). Al calibrar las lecturas del SEM con muestras de suelo, es pues inevitable un error experimental tanto mayor cuanto más difieran las conductivdades de las capas no desmuestradas con respecto a las analizadas en laboratorio. Obsérvese que EMH es más apropiada que EMV para medir conductividades en la zona radicular de los cultivos porque es menos sensible a las capas profundas.

EI SEM está diseñado para que, en el caso indicado de salinidad constante con la profundidad, dé las lecturas EMH=EMV; sin embargo, lo más frecuente es encontrar diferencias entre ambas lecturas. Por este motivo, algunos autores (Corwin y Rhoades, 1982; Slavich y Petterson, 1992) refieren la conductividad ofrecida por el sensor no a EMH o EMV directamente, sino a une combinación lineal de ambas escogida según el tipo de perfil salino. En este trabajo se contemplan tres tipos de perfil: regular, cuan- do la CEe aumenta en profundidad; invertido, cuando la CEe disminuye en profundidad; y uniforme cuando la CEe es constante en profundidad. Obsérvese en [2] que, conocido el perfil salino, pueden deducirse EMH y EMV, pero un determinado par EMH y EMV puede deberse a distintos perfiles. Pese a ello, Cook y Walker (1992) han empleado un método matemático para obtener combinaciones lineales de funciones de respuesta que minimizan la contribución de las capas de suelo fuera del intervalo de interés; así, podrian emplearse las funciones [1] o varias otras si se dispone de distintos sensores. Los propios autores advierten que minimizar la respuesta en un dominio de profundidades no significa insensibilizar el aparato o aparatos en él.

A los problemas derivados de la heterogeneidad vertical y horizontal del suelo se añade el que el compartamiento del SEM depende sensiblemente de la conductividad eléctrica. Así, el método de Cook y Walker (1992) exige condiciones de bajo número de inducción, es decir, salinidades moderadas o bajas. Para evaluar la desviación de la linealidad fuera de dichas condiciones se ha utilizado la función que gobierna la respuesta del EM-38 (McNeill, 1980a):

$$
\sigma_{a}=\frac{4}{\omega \mu_{0} s^{2}}\left(\frac{H_{s}}{H_{p}}\right)_{\substack{\text { en componente } \\ \text { en cutura }}}
$$

donde $\sigma_{\mathrm{a}}$ es la conductividad eléctrica de un horizonte suficientemente grueso (supuesto homogéneo), $\mathrm{H}_{\mathrm{s}} / \mathrm{H}_{\mathrm{p}}$ es la relación entre los campos magnéticos secundario (originado en el medio conductivo) y primario (transmisión directa desde la bobina emisora) en la bobina receptora, $\omega$ es la frecuencia angular de la onda emitida, $s$ es la distancia entre bobinas y $\mu_{0}$ la permeabilidad magnética del vacio. A partir de [3] se han desarrollado expresiones explicitas sobre la dependencia de EMH y EMV con la conductividad $\sigma$ y con la permeabilidad magnética relativa del suelo $\mu_{r}$

Además, las técnicas electromagnéticas requieren calibrar el sensor para cada suelo. La calibración relaciona dos conductividades eléctricas obtenidas por medios diferentes. Así, cuando se calibra con CEe, además de destruir la estructura del suelo, se añade agua y pueden liberarse iones que no contribuyeron a la lectura del sensor. Por otro lado, las muestras se extraen con barrena recogiendo un volumen de suelo mucho menor que el interceptado por el SEM. Este problema se agrava cuanto mayor es la heteroge- 
neidad edáfica, situación típica de los suelos salinos que tienden a presentar rodales.

La respuesta del SEM a la salinidad depende del contenido de humedad del suelo. Ello impone la limitación, incluso para un mismo suelo, de hacer las medidas cuando la humedad edáfica está dentro del intervalo en que la respuesta puede considerarse constante (McKenzie, 1989), apreciable a simple vista. Se asume que el efecto sobre la calibración de otros factores como la mineralogía, la estructura y la composición iónica de la solución de suelo, no varía en el plazo de tres años aquí estudiado.

\section{MATERIALES Y MÉTODOS}

Los datos experimentales se han obtenido de una parcela de 0,5 ha. Las lecturas EMH y EMV se tomaron en mayo de 1988, enero de 1989 y abril de 1990 sobre una misma red de 52 puntos separados $10 \mathrm{~m}$ a lo largo y ancho de la parcela. Se eligieron fechas unos días después de un riego o lluvia intensa, para que el suelo contuviera el agua retenida en equilibrio gravitatorio (capacidad de campo). En 1988 y 1989 se ajustaba el cero del EM-38 sobre un medio de conductividad nula. En 1990 se dispuso de dos sensores y se probó otro método de puesta a cero propuesto por McNeill (1980b). Por este motivo, en ese año los datos se obtuvieron de tres modos diferentes: sensor 1 con la $1^{\text {a }}$ puesta a cero, designados como "1990a"; sensor 2 con la 1a puesta a cero "1990b"; y sensor 2 con la $2^{a}$ puesta a cero "1990c". En total se tienen cinco conjuntos de datos; los tres de 1990, por ser de una misma fecha y parcela, han servido para estudiar la autoconsistencia de las medidas del SEM.

Para que el SEM sea de utilidad práctica, se consideró que el número de puntos de extracción de muestra de suelo no debe sobrepasar el $30 \%$ de los puntos de lectura. Por ello, en cada año se escogió el azar un número reducido $\mathrm{N}$ (Tabla I) de los puntos de lectura para extraer con barrena muestras de suelo. Se llegó hasta la profundidad de $1 \mathrm{~m}$ que se deseaba calibrar, extrayéndolas a intervalos de $25 \mathrm{~cm}$ y verificando para cada una que estuviera cercano a la capacidad de campo. Se determinó en laboratorio la conductividad eléctrica el extracto de saturación (CEe), la del extracto $1: 5$ (CE5) y la humedad de saturación (Hs) en cada una de las muestras. El plan muestreo fue más que suficiente para deducir la salinidad media de la parcela (Díaz y Herrero, 1992).

Utilizando las coordenadas de los puntos de lecturas del SEM se obtuvieron representaciones tridimensionales de EMH y EMV en las tres fechas. Para ello se utilizó el PROC G3GRID del paquete estadístico SAS (SAS Institute Inc, 1988), que interpola dividiendo la parcela en un mosaico de triángulos con vértices en los puntos de lectura, y ajusta un polinomio bivariante de quinto grado en cada triángulo. El suavizado se logra con una estimación de las derivadas primeras y segundas de la función de ajuste basada en los tres puntos más cercanos a cada vértice. Los cálculos estadísticos se llevaron a cabo también por los procedimientos del SAS (SAS Institute Inc, 1988) de regre-

Tabla I. Calibraciones del EM-38 con N puntos de muestreo de CEe hasta $1 \mathrm{~m}$ de profundidad en tres años consecutivos, con tres lecturas en el último, sobre una misma parcela. Se presentan tres métodos: regresión simple, $\mathrm{RS}$ (CEe = $\left.a_{1} E M H+a_{2}\right)$; regresión múltiple, $R M\left(C E e=b_{1} E M H+b_{2} E M V+b_{3}\right)$; y regresión logarítmica $R L\left(C E e=c_{1} \ln E M H+c_{2}\right)$. Entre paréntesis figura el error típico de las estimas.

\begin{tabular}{|c|c|c|c|c|c|}
\hline & 1988 & 1989 & $1990 a$ & $1990 b$ & $1990 \mathrm{c}$ \\
\hline$N$ & 9 & 11 & 16 & 16 & 16 \\
\hline \multicolumn{6}{|l|}{ RS } \\
\hline$a_{1}$ & $5,4(0,9)$ & $3,3(0,9)$ & $5,6(1,0)$ & $5,3(1,0)$ & $5,1(1,0)$ \\
\hline$a_{2}$ & $0,4(1,4)$ & $1,6(1,5)$ & $0,4(1,2)$ & $1,1(1,1)$ & $1,3(1,1)$ \\
\hline $\mathrm{R}^{2}$ & 0,83 & 0,62 & 0,70 & 0,67 & 0,66 \\
\hline \multicolumn{6}{|l|}{ RM } \\
\hline$b_{1}$ & $5,6(2,0)$ & $4,1(3,0)$ & $5,9(1,1)$ & $5,7(1,2)$ & $5,4(1,1)$ \\
\hline$b_{2}$ & $-0,2(2,0)$ & $-0,8(2,9)$ & $-0,6(1,1)$ & $-0,8(1,2)$ & $-0,7(1,1)$ \\
\hline$b_{3}$ & $0,5(1,6)$ & $1,6(1,6)$ & $0,7(1,1)$ & $1,5(1,2)$ & $1,7(1,3)$ \\
\hline $\mathrm{R}^{2}$ & 0,83 & 0,63 & 0,70 & 0,68 & 0,66 \\
\hline \multicolumn{6}{|l|}{ RL } \\
\hline$c_{1}$ & $7,8(0,9)$ & $5,8(1,3)$ & $5,6(0,9)$ & $4,9(0,8)$ & $4,6(0,8)$ \\
\hline $\mathrm{c}_{2}$ & $5,8(0,4)$ & $4,6(0,8)$ & $7,3(0,3)$ & $7,6(0,3)$ & $7,6(0,3)$ \\
\hline$R^{2}$ & 0,91 & 0,69 & 0,75 & 0,73 & 0,72 \\
\hline
\end{tabular}


sión simple y múltiple, análisis por componentes principales, procedimientos no lineales y estadística de base.

\section{RESULTADOS Y DISCUSIÓN}

\section{Calibraciones}

Las calibraciones de las lecturas del SEM con la CEe desde 0 hasta $100 \mathrm{~cm}$ (Tabla I) fueron obtenidas utilizando cada uno de los cinco conjuntos de datos. Los términos independientes de la regresión simple (RS) y la múltiple (RM) no pueden interpretarse como una $\mathrm{CEe}$ residual porque no son significativamente distintos de cero en ningún caso. Además las calibraciones de 1990, que comparten las mismas muestras de pasta saturada, presentan diferencias notables entre sus términos independientes. Todo ello induce a banalizar unos términos de tan escaso valor estadístico.

Como sugieren las representaciones de las lecturas EMH y EMV en la parcela (fig 1), la zona de mayor variabilidad de las lecturas queda, aproximadamente, en el rincón de coordenadas rectangulares $X$ e $Y$ entre 0 y $30 \mathrm{~m}$. Este rincón coincide con la entrada de agua a la parcela. Al eliminar de cada juste las muestras correspondientes se encuentra una mejora sustancial en el $\mathrm{R}^{2}$ de las regresiones (Tabla II). Este hecho es el núcleo del problema metodológico de la calibración del SEM: si se respeta la elección al azar de

Fig 1. Representación de las lecturas del SEM en la parcela de estudio. Las superficies se han obtenido por interpolación de los datos experimentales Pueden compararse los mapas de EMH y EMV en 1988,1989 y 1990 a.
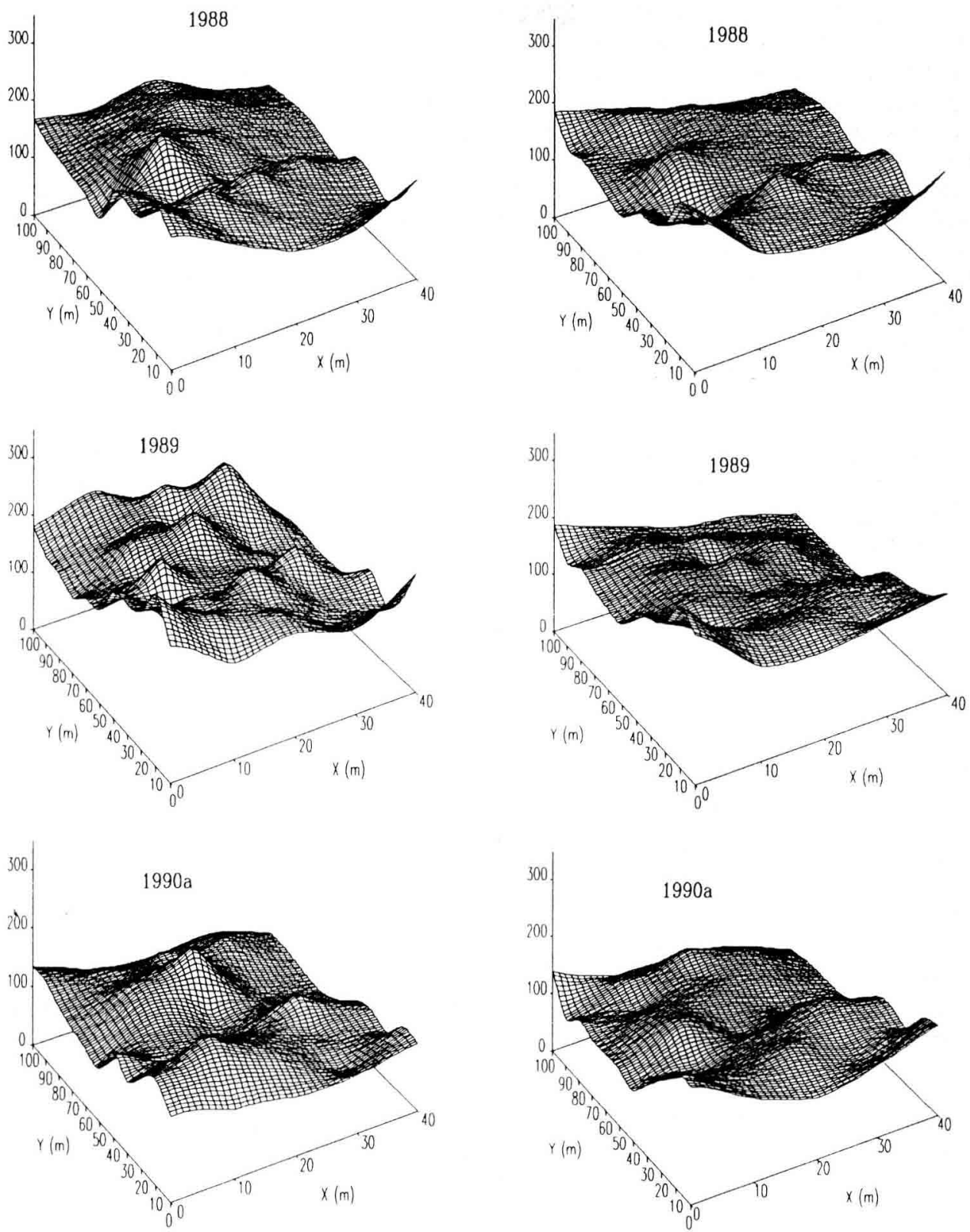
los puntos de barrena, de donde se obtiene CEe, debe tenerse en cuenta que tres o cuatro puntos de muestreo pueden hacer variar notablemente los coeficentes de la calibración. Como se comprueba en la Tabla II, los coeficientes de EMH para 1989 y 1990a difieren considerablemente de los de la Tabla 1, obtenidos sin eliminar ningún punto. Esta dependencia de los coeficientes con la selección de puntos de muestreo justifica el uso de una calibración sencilla, como es el método RS, a la par que desvirtúa interpretaciones más deterministas de las ecuaciones obtenidas.

Puede observarse en la Tabla I que la incorporación de EMV a las calibraciones (método $\mathrm{RM}$ ) mejora ligeramente las $R^{2}$ en dos calibraciones. Sin embargo, coincidiendo con Lesch y Rhoades (1992), se encuentra una alta colinealidad entre EMH y EMV, y el coeficiente de EMV nunca es significativamente distinto de cero; todo ello pone en entredicho el método RM. Estos autores solventan el inconveniente definiendo nuevas variables regresoras $y$ tomando lecturas de microelevación, lo que no es aplicable en parcelas niveladas como las del presente trabajo.
Además, la incorporación de nuevas variables va en contra de la sencillez deseable para usos rutinarios en campo.

Cuando dentro de la misma parcela exista suficiente variabilidad de perfiles, EMH y EMV podrán ser fuentes independientes de información. No parece ser éste el caso en la parcela prospectada y, de hecho, un análisis por componentes principales (Tabla III) de EMH, EMV, $\mathrm{CEe}, \mathrm{CE} 5$ y $\mathrm{Hs}$ en los cinco conjuntos de datos, muestra que la variabilidad se ciñe a un solo eje principal, bien correlacionado con EMH, lo que desaconseja tomar EMV como nueva fuente de información. En todas las fechas, la variable de laboratorio más representativa de la variabilidad total es CEe, mientras que EMH lo es para las lecturas del SEM, justificando una calibración por el método RS.

Cook y Walker (1992), suponiendo una respuesta lineal del SEM a la CE del suelo (McNeill, 1980a), priman la información acerca de un determinado horizonte combinando linealmente funciones de respuesta. En el presente trabajo se procede a la inversa, es decir, una vez obviado el término independiente, las ecuaciones

Tabla II. Calibraciones por regresión simple $\mathrm{RS}\left(\mathrm{CEe}=\mathrm{a}_{1} \mathrm{EMH}+\mathrm{a}_{2}\right)$ obtenidas eliminando los puntos de la zona de mayor salinidad de la parcela. Entre paréntesis figura el error típico de las estimas.

$\begin{array}{cccccc} & 1988 & 1989 & 1990 a & 1990 b & 1990 c \\ N & 6 & 7 & 13 & 13 & 13 \\ & & & & & \\ a_{1} & 6,0(1,1) & 5,7(1,5) & 6,1(0,8) & 5,8(0,8) & 5,6(0,8) \\ a_{2} & -0,27(1,5) & -1,4(2,1) & 0,1(1,0) & 0,9(0,9) & 1,1(0,9) \\ R^{2} & 0,85 & 0,72 & 0,83 & 0,81 & 0,80\end{array}$

Tabla III. Análisis por componentes principales en los cinco conjuntos de datos del estudio. Se muestran las correlaciones entre el eje asociado al autovalor de mayor valor absoluto $(\lambda)$ y las variables EMH, EMV, CEe (conductividad eléctrica del extracto de la pasta saturada), CE5 (conductividad eléctrica del extracto 1:5) y Hs (humedad de saturación). También se muestra la varianza explicada por cada eje factorial (var).

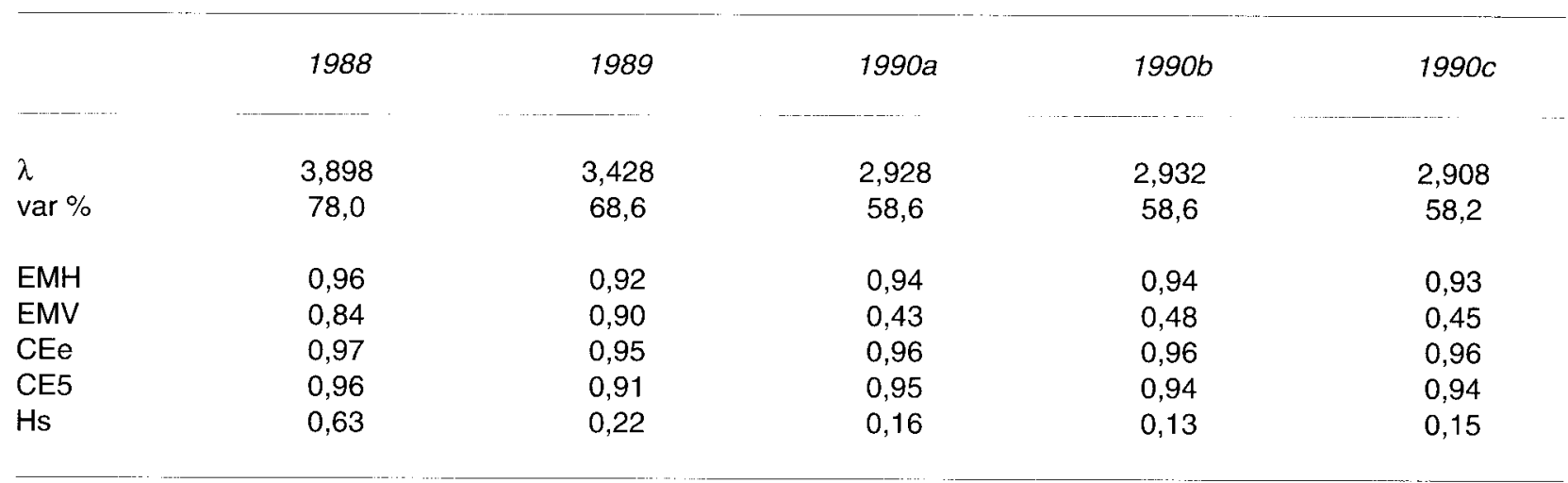


del método RM (Tabla I) se interpretan como precedentes de combinaciones lineales de las funciones [1]. Un algoritmo de calibración CEe = $a_{1} E M H+a_{2} E M V$ puede asignarse a une función de respuesta normalizada $\Phi_{\text {SEM }}=b_{1} \Phi H+b_{2} \Phi v$, donde $b_{1}=a_{1} /\left(a_{1}+a_{2}\right), b_{2}=a_{2} /\left(a_{1}+a_{2}\right)$, de la cual se espera que informe sobre qué capas han contribuido más al ajuste experimental. A partir de la contribución de todo el volumen explorado por el SEM, el ajuste RM está explicando la conductividad CEe hasta $1 \mathrm{~m}$. Cuando predominan los perfiles regulares, la lectura $E M H$ debe resultar más apropiada para explicar la conductividad de la capa de muestreo; si predominan los invertidos, debe ser más apropiada EMV. Así lo apuntan en la Tabla IV muestras calibraciones del año 1990, en que se dispuso de puntos suficientes de muestreo para poder seleccionar grupos de puntos con marcado carácter regular o invertido según las muestras de laboratorio. Puesto que, como se ha constatado (Tabla I), algunos coeficientes de nuestro ajuste RM no son significativos, se ha aplicado el método descrito a los datos de Slavich (1990) obteniendo los resultados de la Tabla IV. Se observa (Fig 2) que
$\Phi_{\text {SEM }}$ es de mayor alcance en perfiles invertidos, para los que EMV no ha tenido una contribución importante, y al revés para los perfiles regulares. La Tabla IV muestra también cómo el coeficiente $b_{1}$ que acompaña a $\Phi_{H}$ disminuye su valor conforme se pasa de un perfil globalmente regular a uno invertido, y al revés en el caso del coeficiente de $\Phi_{V}$.

Esta interpretación del ajuste RM hasta $1 \mathrm{~m}$ permite una caracterización sencilla de los perfiles una vez obtenidos los coeficientes $b_{1}$ y $b_{2}$ :

$\mathrm{b}_{1}>\mathrm{b}_{2} \rightarrow$ Regulares

$b_{1} \approx b_{2} \rightarrow$ Mixtos

$b_{1}<b_{2} \rightarrow$ Invertidos

Esta caracterización coincide con los resultados de Slavich (1990) de la Tabla IV, que se basan en la simulación de un número grande de perfiles hasta $5 \mathrm{~m}$ de profundidad y su calibración hasta $1 \mathrm{~m}$. En dicha Tabla no se encuentra la misma evidencia con los datos del presente trabajo, porque los perfiles se han clasificado como regulares o invertidos a partir de los datos

Tabla IV. Calibraciones normalizadas $\Phi_{\mathrm{SEM}}=b_{1} \Phi_{\mathrm{H}}+\mathrm{b}_{2} \Phi_{\mathrm{V}}\left(\mathrm{b}_{1}+\mathrm{b}_{2}=1\right)$ sobre los datos de 1990a y sobre los resultados de Slavich (1990) para el mismo intervalo de profundidad $(1 \mathrm{~m})$. Los coeficientes se corresponden con los gráficos de la Figure 2.

\begin{tabular}{lcccccc} 
& \multicolumn{3}{c}{ 1990a } & \multicolumn{3}{c}{ Slavich 1990} \\
Tipo & $b_{1}$ & $b_{2}$ & $R^{2}$ & $b_{1}$ & $b_{2}$ & $R^{2}$ \\
& & & & & & \\
Regulares & 2,08 & $-1,08$ & 0,82 & 1,24 & $-0,24$ & 0,99 \\
Todos & 0,90 & 0,10 & 0,70 & 0,57 & 0,43 & 0,97 \\
Invertidos & 0,78 & 0,22 & 0,47 & 0,41 & 0,59 & 1,00
\end{tabular}
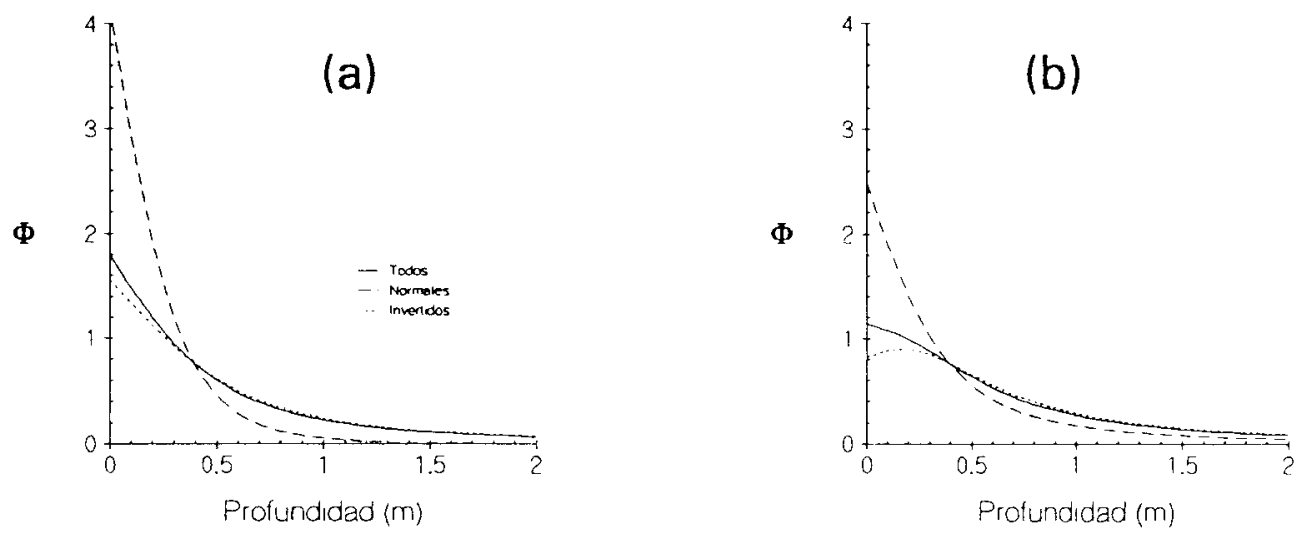

Fig 2. Funciones de respuesta $\Phi$ del EM-38 basadas en la calibración hasta $1 \mathrm{~m}$ de profundidad por regresión múltiple. Las tres curvas de (a) corresponden a una selección de perfiles de 1990 marcadamente regulares o invertidos según las muestras de CEe, y a todo el muestreo. En (b) se da el resultado obtenido a partir de los datos de Slavich (1992) con un número grande de perfiles salinos simulados. 


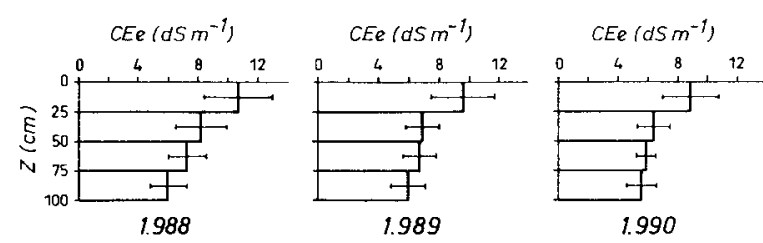

Fig 3. CEe promedio y su desviación típica, a intervalos de profundidad (z) de $25 \mathrm{~cm}$, en las muestras de suelo de 1988, 1989 y 1990

de desmuestre hasta $1 \mathrm{~m}$ (figura 3 ), desconociéndose el resto del perfil, aunque la calibración también es hasta $1 \mathrm{~m}$. El método propuesto para identificar perfiles a partir de los coeficientes de una calibración RM es sencillo y parece ser útil, en especial cuando la calibración RM sea estadísticamente significativa. La aplicación de este método a otras condiciones y suelos permitirá evaluar su validez general.

En nuestro caso, de la Tabla IV se interpreta que hay mayoria de perfiles de tipo regular o cercano a uniforme. Esto está de acuerdo con la alta correlación entre EMH y EMV y es compatible con la Figura 3 porque el tipo de perfil se refiere al alcance del sensor y no sólo al metro superficial, objeto de la calibración, y que se representa en dicha Figura. Además, como en los ajustes RM la lectura EMV aporta muy poca información (Tabla I), cabe interpretar que la variabilidad punto a punto de la capa desmuestrada supera a la de las inferiores, menos afectadas por el manejo agrícola. En este sentido, se encuentra que el coeficiente de variación para las lecturas EMH es mayor que el de EMV en las tres tandas de lecturas de 1990; en promedio, el coeficiente de variación para EMH es del $29,2 \%$ y para EMV del $27,1 \%$. Ello es más significativo en tanto que dicha capa sólo contribuye un $20 \%$ más a EMH que a EMV, según muestra la integración de las funciones [1] (McNeill, 1980a). La mayor variabilidad de EMH queda ilustrada en la Figura 1, donde las lecturas EMV dan mapas con mayor suavizado que las lecturas EMH.

Se plantea el inconveniente de que una salinidad homogénea puede ajustarse en teoría a funciones de respuesta cualesquiera, $p$. ej. $\Phi_{\mathrm{SEM}}=$ $\Phi_{H} ; \Phi_{\mathrm{SEM}}=\Phi_{\mathrm{V}} ; \Phi_{\mathrm{SEM}}=0,5 \Phi_{H}+0,5 \Phi_{\mathrm{V}} \cdot \operatorname{Sin}$ embargo, en este caso es preferible un ajuste por regresión simple, con EMH o EMV dependiendo del alcance deseado para la prospección $y$ teniendo en cuenta la profundidad de las muestras de suelo disponibles para la calibración.

Las calibraciones que hacen uso de la transformación logarítmica persiguen estabilizar la varianza cuando se encuentra una proporcionali- dad entre la desviación típica y la media de la magnitud. Para interpretar estas calibraciones (Tabla I) debe señalarse que, en el caso del SEM, dicha proporcionalidad se puede identificar en dos niveles distintos.

Por un lado, la salinidad es mucho más variable en suelos salinos que en no salinos, como lo denota incluso visualmente la aparición de eflorescencias en rodales. Además debe reseñarse que estos corros suelen tener tamaños métricos, lo que exacerba las diferencias de variabilidad de las lecturas del EM-38 frente a las de barrena. Al respecto, Hendrickx et al (1992) encuentran que la media de EMH es proporcional a la desviación típica por un factor de $1 / 3$ (algo más de $1 / 4$ en este trabajo), basándose en 351 parcelas.

Por otro lado, la resolución de las lecturas del sensor es de aproximadamente un 3\% debido al proceso analógico de la medida, que incorpora sistemáticamente una cierta proporcionalidad entre la medida y su error. La Tabla $V$ muestra el resultado de comparar los tres conjuntos de lecturas de 1990. Obsérvese que los conjuntos más parecidos son los correspondientes a un mismo detector; todos ellos constan de 51 lecturas y las diferencias entre medias se encuentran en torno a un $7 \%$ para las combinaciones a-b, a-c (distintos detectores) y en torno a un $3 \%$ para el mejor caso b-c (mismo detector).

La mayor dispersión de los datos correspondientes a conductividades mayores podría compensarse primando la toma de muestras de suelo en las zonas donde las lecturas del sensor son mayores. En el futuro deberán investigarse los criterios para tal compensación, y para optimizar el muestreo, como recientemente han hecho Lesch et al (1995).

Según se ha comentado, a través de la naturaleza del suelo y de un condicionante técnico puede aparecer en el ajuste CEe-SEM una proporcionalidad entre las medias y sus desviaciones típicas, que suele implicar una relación no lineal entre las variables. La literatura sobre el SEM no suele destacar el carácter lineal o no de las calibraciones, de manera que la transformación logarítmica se efectúa en base a argumentos estadísticos. Por ello, el método $\mathrm{RL}$ de la Tabla I debe interpretarse con cautela atendiendo no sólo a la mejora en $\mathrm{R}^{2}$, sino también a su dependencia con el recorrido de los valores de calibrado, mayor que en los métodos lineales. Además, se ha comparado la calibración $R L$ con la calibración RS calculando la distancia (dS/m) media entre sus funciones en el recorrido de los 
valores de calibración. Para las distintas fechas de calibración de este trabajo, dicha distancia media se encuentra entre 0,42 y $1,64 \mathrm{dS} / \mathrm{m}$, siempre por debajo de los errores de las estimas de CEe en función de los errores de los parámetros de la calibración.

\section{Repuesta del SEM}

A partir de las notas técnicas del SEM (McNeill, 1980) se han encontrado las expresiones matemáticas que relacionan las lecturas del aparato con la conductividad eléctrica $\sigma$ del medio expresada en $\mathrm{S} / \mathrm{m}$, su permeabilidad magnética $\mu$ y la frecuencia angular $\omega$ de la onda emitida:

$E M H=\frac{4}{\omega \pi} \operatorname{lm}\left\{2\left[1-\frac{3}{y^{2}}+\left(3+3 y+y^{2}\right) \frac{e^{-y}}{y^{2}}\right]\right\}[4 a]$

$$
E M V=\frac{4}{\omega \pi} \operatorname{lm}\left\{\frac{2}{y^{2}}\left[9-\left(9+9 y+4 y^{2}+y^{3}\right) e^{-y}\right]\right\}[4 b]
$$

donde $\mathrm{Im}$ es el operador que selecciona la parte imaginaria de su argumento y donde:

$$
y=\sqrt{\omega \mu \sigma \cdot e} i \frac{\pi}{4}
$$

En las Figuras $4 a$ y $4 b$ se representan las funciones [4] en el recorrido de valores que puede dar el EM-38, y se observa que EMV se desvía más de la linealidad que EMH. A su vez, en la Tabla VI se dan los listados de varios puntos de los gráficos que pueden servir de ayuda para corregir las lecturas del sensor. En dicha Tabla puede observarse que la corrección no es importante para lecturas del sensor moderadas 0 bajas, orientativamente para $\mathrm{EMH}<300, \mathrm{EMV}<$ 200. Los datos presentados por Corwin y

Tabla V. Comparación entre las lecturas efectuadas en 1990 con dos sensores EM-38 y según los dos métodos de puesta a cero propuestos por Geonics Ltd. Se comparan 51 lecturas en posición horizontal (EMH) y vertical (EMV) en los casos: entre detectores con la misma puesta a cero (a-b); entre detectores con distintas puestas a cero (a-c); el mismo detector con distintas puestas a cero (b-c).

$N^{\circ}$ de lecturas coincidentes

\section{Diferencia absoluta entre medias ( $\mathrm{mmho} / \mathrm{m}$ )}

$N^{\circ}$ de lecturas diferentes (> 10\%)
$R^{2}$

0,98
0,98
0,99
0,97
0,97
0,99

0,98

$18(35 \%)$

$0(0 \%)$

0,99

$15(29 \%)$

$8(16 \%)$

0,97

$2(4 \%)$
EMVa-EMVc

$E M V b-E M V_{C}$
8
3,9

6,0
0,2
6,8
3,9
2,9

Tabla VI. Listado de algunos puntos correspondientes a las figuras $4 \mathrm{a}$ y $4 \mathrm{~b}$. Debido a que el EM-38 ofrece las lecturas en $\mathrm{mmho} / \mathrm{m}=(\mathrm{dS} / \mathrm{m}) .100$, la tabla se da en dichas unidades.

$\sigma$

$E M H$

\section{0}

61

124

163

185

202

263

316

377

416

474

488
EMV

$\sigma$

$E M H$

517

566

579

600

688

754

789

804

895

1000

1132

1249

EMV

321

351

395

405
472

514

525

544

619

675

704

717

792

879

987

1082
427

464

472

488

550

596

619

630

689

760

843

915 


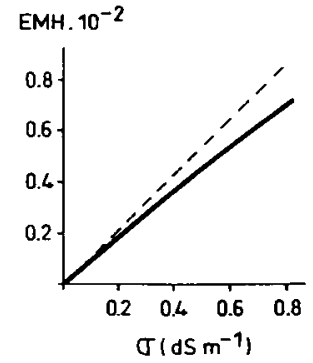

(a)

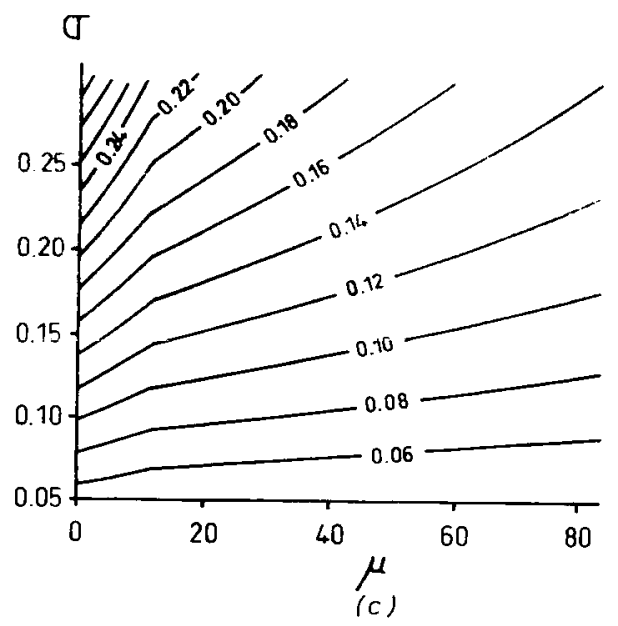

Fig 4. Variación teórica de las lecturas EMH (a) y EMV (b) del sensor electromagnético frente a la conductividad de un medio homogéneo. Se observa que EMV se aleja más que EMH del comportamiento ideal representado por las líneas continuas. En (c) se aprecian las líneas de lectura EMH constante en función tanto de la conductividad eléctrica $\sigma$ del medio como de la permeabilidad magnética relativa $\mu$.

Rhoades (1990) muestran la misma tendencia que la Tabla VI, así como un acuerdo numérico aceptable.

Las funciones [4] son incómodas de manipular tratándose de funciones de variable compleja, dado que el interés se centra más bien en conocer sus inversas, es decir, los algoritmos $\sigma=$ $\sigma(E M H)$ y $\sigma=\sigma(E M V)$. Para ello se pueden interpolar los valores de la Tabla VI a las lecturas requeridas, o bien invertir numéricamente una función aproximada para [4] que resulte más manejable. Desarollando las exponenciales de [4] hasta el quinto orden, que se ha encontrado suficiente, en serie de Mc Laurin se obtienen las siguientes funciones reales para las lecturas del EM-38:

$$
E M H=\sigma-\left(-1,586.10^{-3} \sigma^{2,5}+0,1217 \sigma^{1.5}\right)
$$

$$
E M V=\sigma-\left(7,242.10^{-4} \sigma^{-3}-1,586.10^{-3} \sigma^{2,5}+0,2435 \sigma^{1,5}\right)
$$

que son válidas, al igual que [4], cuando $\sigma$ se expresa en $\mathrm{S} / \mathrm{m}$.
Las funciones [4] también se han usado para obtener la respuesta teórica del SEM con la permeabilidad magnética relativa del medio $\mu_{r}=$ $\mu_{\text {medio }} / \mu_{0}$. La Figura $4 \mathrm{c}$, muestra que $\sigma$ y $\mu$ concurren en disminuir la sensibilidad del EM-38. También conviene señalar que, así como las sustancias mayoritarias en el suelo presentan valores $\mu_{r}$ del orden de la unidad, las ferromagnéticas alcanzan valores superiores en varios órdenes de magnitud. Así, un pequeño volumen de materiales ferromagnéticos, aún promediado a todo el volumen explorado por el SEM, podría contribuir a disminuir la lectura apreciablemente. Debe tenerse en cuenta que los materiales ferromagnéticos pueden ser además buenos conductores.

Desde el punto de vista estadístico las lecturas del SEM comportan un sesgo a la baja, tanto mayor cuanto mayor es la lectura. La transformación logaritmica aplicada al valor SEM corrige la desviación de la linealidad pero no el sesgo. Así, en Lesch y Rhoades (1992) aparecen conjuntos grandes de datos donde las lecturas EMH disminuyen su proporcionalidad respecto a CEe.

\section{CONCLUSIONES}

En ausencia de una justificación determinista para usar relaciones no lineales entre las lecturas del SEM y CEe, se recomiendan las calibraciones lineales. En efecto, el método RS es el más sencillo y no aporta menos información que el RL en estas parcelas. Por otro lado, el método RM puede informar acerca del perfil salino.

La única no linealidad entre la respuesta del SEM y la conductividad del medio explicable físicamente se ha encontrado en la propia definición de las lecturas del SEM, pero para lecturas del sensor moderadas o bajas ello es irrelevante. Si las lecturas del SEM son elevadas, la proporción entre ellas y la conductividad del medio cambia, y lo hace más acusadamente para EMV que para EMH. Este efecto puede corregirse en base a las características técnicas del aparato y retornar al uso de métodos lineales de calibración.

\section{AGRADECIMIENTOS}

Este trabajo fue financiado por INIA (Ministerio de Agricultura, Pesca y Alimentación). 


\section{REFERENCIAS}

Ayers RS, Westcot DW (1987) La calidad del agua en la agricultura. Estudio FAO riego y drenaje 29, rev 1, Roma, $174 \mathrm{p}$

Cook PG, Walker GR (1992) Depth profiles of electrical conductivity from linear combinations of electromagnetic induction measurements. Soil Sci Soc Am $J 56,1015-1022$

Corwin DL, Rhoades JD (1982) An improved technique for determining soil electrical conductivitydepth relations from above-ground electromagnetic measurements. Soil Sci Soc Am J 46, 517-520

Corwin DL, Rhoades JD (1984) Measurement of inverted electrical conductivity profiles using electromagnetic induction. Soil Sci Soc Am J 48, 288291

Corwin DL, Rhoades JD (1990) Establishing soil electrical conductivity-depth relations from electromagnetic induction measurements. Commun in Soil Sci Plant Anal 21 (11\&12), 861-901

Díaz L, Herrero J (1992) Salinity estimates in irrigated soils using electromagnetic induction. Soil Science 154, 151-157

Hendrickx JMH, Baerends B, Raza ZI, Sadig M, Akram Chaudry M (1992) Soil salinity assessment by electromagnetic induction of irrigated land. Soil Sci Soc Am J 56, 1933-1941
Lesch MS, Rhoades JD (1992) Mapping soil salinity using calibrated electromagnetic measurements. Soil Sci Soc Am J 56, 540-548

Lesch MS, Strauss DJ, Rhoades JD (1995) Spatial prediction of soil salinity using electromagnetic induction techniques. 2. An efficient spatial sampling algorithm suitable for multiple linear regression model identification and estimation. Water Resour Res 31, 25-32

McKenzie RC, Chomistek W, Clark NF (1989) Conversion of electromagnetic unductance readings to saturated paste extract values in soils for different temperature, texture, and moisture conditions. Can J Soil Sci 69, 25-32

McNeill JD (1980a) Survey interpretation techniques EM-38. TN-6 Geonics Ltd

McNeill JD (1980b) Survey interpretation techniques EM-38. TN-9 Geonics Ltd

Royo A, Aragüés R, Quílez D (1991) Descripción y evaluación de cuatro modelos de respuesta de cultivares de cebada a la salinidad. Invest Agr Prot Veg 6(3), 319-330

SAS Institute Inc (1988) SAS/GRAPH User's guide, Release 6.03, SAS Institute Inc Cary, NC

Slavich PG (1990) Determining ECa-depth profiles from electromagnetic induction measurements. Aust $J$ Soil Res 28, 443-452

Slavich PG, Petterson GH (1990) Estimating average rootzone salinity from electromagnetic induction (EM38) measurements. Aust J Soil Res 28, 452-463 